\title{
Evolution of the criminological profile of the Islamic terrorist. The European response for the prevention of the new jihadist criminal
}

Evoluzione del profilo criminologico del terrorista islamico. La risposta europea per la prevenzione al nuovo criminale jihadista Evolución del perfil criminológico del terrorista islámico. La respuesta europea para evitar el nuevo criminal yihadista

\author{
Maria Campioni, ${ }^{1}$ Vincenzo M. Mastronardi ${ }^{2}$ \\ ${ }^{1}$ Mediatrice linguistica, dottoressa in Criminalità, Investigazione e Sicurezza Internazionale; ${ }^{2}$ Psichiatra, Criminologo \\ Clinico, Sapienza Università di Roma; Docente di Teoria della Devianza e Crimninogenesi, Università degli Studi \\ Internazionali di Roma, Italy
}

\begin{abstract}
The threat of the new century brings the face of men and women, driven to subversion by an extreme idea, because of misinterpretation. They are an army fighting an asymmetrical conflict at while international relations have changed. Jihad, which is why Al Qaeda has shed blood for years, has now evolved, adapting itself to society, instrumentalizing the religious concept, the cornerstone of Islamist values. But who is the criminal you are looking for? How does he move? And how recognize him? These questions have been answered by analyzing the jihadist profiles, already available and the events of the last few years in order to have medium-long term projections. Fulcrum of the evolution of the jihadist soldier is the birth of the Isis and the media propaganda. The greatest attention is paid to the younger ones, as they are more influential on virtual platforms and migration flows. Europe recognizes the risk of radicalization on its territory, already victim of numerous attacks, and although it does not yet have a common governance of action against this threat, it has adopted prevention plans consisting of integration, information, re-education and democracy. From Italy comes the scientific study, operating on three levels, which suggests the method of action for an effective prevention. The objective is to make more fluent cooperation between criminology and counteraction by the combination of the elements of this paper.
\end{abstract}

\section{RIASSUNTO}

La minaccia del nuovo secolo porta il volto di uomini e donne, spinti all'eversione da un'idea estrema, frutto della mal interpretazione. Essi sono un esercito che combatte un conflitto asimmetrico in un'epoca in cui le relazioni internazionali sono cambiate. La Jihad, concetto per cui Al Qaeda ha versato del sangue per anni, ora si è evoluta, adattandosi alla società, strumentalizzando il concetto religioso, cardine dei valori islamisti. Ma chi è il criminale che si sta cercando? Come si muove? E quali sono le caratteristiche con cui poterlo riconoscere? A questi quesiti si è voluto rispondere analizzando i profili jihadisti già a disposizione e gli accadimenti degli ultimi anni per poter avere delle proiezioni di medio/lungo termine. Fulcro dell'evoluzione del soldato jihadista è la nascita dell'Isis e la propaganda mediatica. L'attenzione maggiore è rivolta ai più giovani, in quanto più influenti sulle piattaforme virtuali e ai flussi migratori. L'Europa riconosce il rischio di radicalizzazione sul proprio territorio, già vittima di numerosi attentati, e pur non avendo ancora una comune governance di azione di contrasto alla minaccia ha adottato dei piani di prevenzione costituiti da: integrazione, informazione, rieducazione e democrazia. Arriva dall'Italia lo studio scientifico, operante su tre livelli che suggerisce il metodo di azione per una efficace prevenzione. L'obiettivo è quello di rendere fluida la cooperazione tra criminologia e azione di contrasto con l'unione degli elementi di studio.

\section{RESUMEN}

La amenaza del nuevo siglo trae consigo el rostro de hombres y mujeres, impulsados a la subversión por una idea extrema, resultado de una mala interpretation. Ellos son un ejército que lucha contra un conflicto asimétrico en un momento en que las relaciones internacionales han cambiado. La Jihad, un concepto por el que Al Qaeda ha derramado sangre durante los años, ha evolucionado ahora, adaptándose a la sociedad, instrumentalizando el concepto religioso, la piedra angular de los valores islamistas. ¿Pero quién es el criminal que está buscando? ¿Cómo se mueve? ¿Y cuáles son las características con las que se le reconoce? Estas preguntas se han respondido analizando los perfiles yihadistas ya disponibles y los acontecimientos de los últimos años para tener proyecciones a medio-largo plazo. La evolución del soldado yihadista es el nacimiento de la Isis y la propaganda mediática. Se presta mayor atención a los más jóvenes, ya que son los que más influyen en las plataformas virtuales y en las corrientes migratorias. Europa reconoce el riesgo de radicalización en su territorio, ya víctima de numerosos ataques, y aunque todavía no tiene una gobernanza común de acción contra la amenaza, ha adoptado planes de prevención que consisten en: integración, información, reeducación y democracia. De Italia procede el estudio científico, que opera en tres niveles, que sugiere el método de acción para una prevención eficaz. El objetivo es hacer fluir la cooperación entre la criminología y la acción de contrarrestarla con la unión de los dos elementos de este estudio. 


\section{Introduzione}

Il lavoro di tesi qui proposto è frutto dello studio di un fenomeno ancora poco definibile, nonostante le sue origini antiche, qualcosa contro cui si combatte in un'asimmetria bellica sconosciuta al comune paradigma westfaliano1, di cui le società moderne portano il marchio. Il terrorismo esiste nella storia quanto nel presente, scaturito da un'eversione di base, una chiara volontà di violenza e dominanza capace di piegare un popolo con l'arma del terrore. Ne perviene un concetto dal significato astratto e non inquadrabile in un'unica accezione in quanto sia l'emblema della mutevolezza. Il mero atto eversivo trova dimora in un vissuto composto di comportamento e temperamento (noto già ai tempi di Ippocrate e Galeno), prima ancora di essere idea e biforcarsi nella sfera politica o confessionale. Nel caso del terrorismo di matrice religiosa islamica, i due mondi sembrano congiungersi, uniti da quel fondamento ed elemento cardine della cultura islamica che è la Shari'a, la quale traduzione indica un "percorso della fonte", ed è riconducibile ad un significato metafisico: legge di Dio, e ad uno pragmatico: scienza giurisprudenziale. Essa, pertanto, contiene la legge islamica e le sue fonti: il Corano, la Sunna. Per cui, scindere le due componenti vorrebbe dire interferire con la volontà divina, destabilizzando il suo popolo (Ummah). Il terrorista islamista si serve di tali concetti per legittimare le sue azioni violente ed illegali, e l'Isis (Stato Islamico di Siria e Iraq), la più recente evoluzione del terrorismo islamico ne è protagonista. Ciò che ha allarmato gli esperti, rispetto all'integralismo e alla rigida reinterpretazione della Shari'a della cellula Al-Qaeda, sono le diverse ideologie ed insieme di vissuti dei militanti Isis, che sembrano discostare dalle ragioni di fede di cui si fa portavoce. A tal proposito, si è voluto dare un'immagine al criminale emergente in tutte le sue variabili. La creazione del profilo criminologico è resa possibile da uno studio di più fattori, partendo dal punto di vista etimologico e di difficoltà nel dare una nozione di terrorismo, ai primi profili dei fondatori dell'organizzazione, lo scompiglio che la loro morte ha causato tra gli affiliati e le prospettive

\section{Introduzione al fenomeno del terrorismo: dalla morte di Abu Bakr Al Baghdadi alle testimonianze degli ex combattenti Isis}

Lo studio qui presente si propone di racchiudere le problematiche inerenti al terrorismo di matrice islamica, in particolare tenendo in considerazione l'Isis, in quanto sua ultima evoluzione. Nella resa di tale fenomeno è stato necessario esplicare a livello storico la difficoltà nel concepire un'unica accezione di terrorismo. Esso è derivato dal tirannicidio medievale, ha subìto diverse evoluzioni nel tempo: dalle congiure dell'antica civiltà romana, alle guerre religiose del sedicesimo secolo, fino al terrore giacobino messo in atto da Maximilien de Robespierre, ma è solo nel XIX secolo che la violenza e l'eversione si uniscono alle teorie anarchiche russe di stampo nazionalista. Tuttavia, al giorno d'oggi non è ancora possibile dare una definizione unitaria di tale fenomeno, e l'asimmetria bellica che l'Occidente è costretto ad affrontare è il frutto della volontà di prevalere sul popolo mediorientale, imponendo loro di praticare i valori democratici, cambiandone le radici e approfittandosi del disagio economico e sociale delle zone più arretrate. L'Isis nasce nel 2014 assieme al leader autoproclamato Abu Bakr al Baghdadi, nato a Samarra in Iraq nel 1971 e deceduto il 27 ottobre 2019 a seguito di un raid delle forze armate america- ne, con il contributo delle intelligence mondiali. Egli ha sempre dimostrato profondo interesse nello studio del Corano, caratterizzato da un profilo basso ed estremo pragmatismo. La sua morte ha sicuramente destabilizzato l'ordine dell'organizzazione criminale causando una discesa, iniziata alla fine del 2017 col fenomeno del rientro dei combattenti stranieri in Europa (Foreign Fighters). Conoscendo la forte resilienza delle organizzazioni terroristiche islamiste si è voluto pensare a delle ipotesi circa il futuro prossimo del califfato. Mediante l'uso di un quadrante di generazione delle ipotesi, strumento di utilizzo da parte delle forze di intelligence, è stato possibile tracciare un percorso intrecciando due variabili su un piano cartesiano, ponendo a destra le possibilità di risalita dell'Isis e a sinistra il suo declino, quindi avendo come risultato quattro opzioni, rispettivamente positive e negative:

1. Nella prima ipotesi, la morte del leader fa vacillare la fede degli adepti provocando incertezza e diminuzione degli attacchi in Occidente.

2. Alla conferma della morte del leader, un nuovo capo prende le redini del potere rivendicando la morte del predecessore con nuova violenza.

3. La perdita di un punto di riferimento provoca il completo sfaldamento dell'organizzazione trascinandola nel caos.

4. Sentimenti di vendetta portano a maggiori attacchi in Occidente

Impossibile stabilire con certezza quale delle opzioni si presenterà in futuro, ma il passato del califfato, definito più volte instabile, potrebbe tornare ad attaccare seguendo l'effetto fisarmonica che lo contraddistingue da sempre.

La preoccupazione arriva dai campi di concentramento come quello di Al Hol, che possiede il maggior numero di miliziani, donne e bambini sfollati di Mosul (Raqqa), e di prigionieri che tentano il rimpatrio in Europa. Il governo iracheno prevede che di questi ultimi se ne occupi il proprio paese d'origine anziché adottare come soluzione il creare una sede per un tribunale internazionale. L' Europa muove lentamente i passi verso una soluzione unanime, l'assenza di una governance causa risposte variegate e preoccupanti in ogni Stato membro, che si dicono disposti a togliere la cittadinanza a coloro che, partiti per il fronte siriano e disillusi dall'esperienza vogliano tornare in patria. Michelle Bachelet, direttore esecutivo dell'ente delle nazioni unite per l'uguaglianza di genere spinge le fazioni a riprendere i propri connazionali, nonostante il timore delle conseguenze, mentre Jean-Yves le Drian ministro degli esteri francese dichiara nemici i combattenti che hanno lasciato la Francia per combattere nel Daesh. Il senatore Anthony Dworkin nel Consiglio Europeo degli Affari Esteri tenuto il 25 ottobre 2019 si è espresso in favore al ritorno dei combattenti, elencando una serie di punti che porterebbero guadagno per l'Europa, qualora fosse convenisse con le sue proposte:

1. Sarebbe possibile trattare con i rimpatriati

2. Garantire giustizia per le vittime dell'Isis in Europa

3. Rivelare l'identità e le gesta di altri membri e provare la loro colpevolezza in contumacia

4. Studiare i loro profili da vicino per usarli contro attacchi futuri

5. Dividerli per reati specifici

6. Allontanare i superstiti dai campi di detenzione

7. Sostenere i bambini

8. Attivare programmi di rieducazione sociale

9. Cooperare con le SDF irachene.

Essi sono quindi una risorsa da tenere in considerazione, perciò si ritiene utile evitare i ritardi e chiedere un piano d'azione immediato. Al fine di definire il terrorista foreign fighter se ne riportano le principali caratteristiche di profilazione: 
- $\quad$ Sesso maschile $90 \%$

- Età 30 anni

- Nato all'estero $91,2 \%$

- Assenza di famiglia o indottrinamento dalla stessa

- Fanatismo ideologico

- Marocco e Tunisia come principali paesi d'origine

- Nessun particolare interesse d'origine

- Assenza di relazioni sentimentali $60,8 \%$

- Basso livello di istruzione $87,7 \%$

- Disoccupato $34,4 \%$

- Precedenti penali $44 \%$

- Partecipazione a network radicali 55\%

- Legami con altri gruppi radicali europei.

(Ispi online, il profilo del foreign fighter)

Il terrorista ha come bersaglio l'emotività e la paura dell'individuo civile. Ne sconvolge la quotidianità instaurando col terrore un senso di inferiorità.

Sono state raccolte le testimonianze di militanti tornati dal fronte, come Monsef El Mkhayar, partito nel 2015 e catturato dalle SDF curde. Egli si dichiara pentito di essersi arruolato, sostenendo che la sua esperienza è stata deludente.

"Questa è la mia credenza e non la cambierò, ma qui nello Stato Islamico non c'è giustizia"

(El Mkahyar, in Reuter)

Abu Ibrahim, giovane siriano, studioso di informatica scelgie di arruolarsi con Baghdadi per pochi soldi. Nel momento in cui si trova a dover uccidere dei suoi amici egli si tira indietro, impaurito, disilluso e disgustato dalle torture ed umiliazioni inflitte dall' Isis.

La testimonianza arriva anche da donne e bambini (figli dell'Isis), spesso venduti, donne costrette a sorvegliarsi a vicenda, a subire le torture dei mariti in caso di disobbedienza. I bambini seguono una rigida istruzione, fin da piccoli vengono iniziati alle armi e per sempre porteranno il trauma dell'anaffettività. Non c'è distinzione tra loro ed il resto della popolazione, se necessario vengono uccisi senza rimorso.

Le esperienze degli europei (Ebrahim, 26 anni di origine tedesca) differiscono dai nati al fronte, in quanto la volontà di prendere parte all'organizzazione criminale nasce dal sentimento di vendetta causato da una vita mediocre o priva di successi. Davide Grasso, guerriero italiano YPG (unità di protezione popolare della regione curda siriana), racconta la sua esperienza al fronte contro l'esercito dell'Isis e spiega come sia assente anche solo la paura di morire per i militanti.

\section{Cyber terrorismo, la minaccia si estende al mondo virtuale}

Un ritaglio importante è dedicato alla tecnologia e ai networks, i quali da una parte hanno facilitato la quotidianità e dall'altra sono strumento nelle mani della criminalità mondiale. La navigazione sul web e l'investimento nella ricerca in ambiente cyber nasce dall'idea di Wiliam Gibson nel suo romanzo Neuromante, 1984. L'idea mostra al lettore una realtà parallela che sia da trampolino per il futuro dell'uomo, verso uno scambio informativo più efficiente e che non tenga conto di confini e distanze geografiche. Il cyber spazio nasce come bene comune, come piattaforma per mostrarsi al mondo, eppure non è ancora chiara una comune governance al riguardo. Il web è caratterizzato da tre livelli, il primo è quello di comune accesso con normali portali o motori di ricerca, il secondo livello entra nel Deep web, ossia quella parte di internet di difficile accesso e che comprende il $90 \%$ di tutto il web, mentre il Dark web corrisponde al $6 \%$ del web totale. Per accedere a questi luoghi oscuri del web esistono dei server: Tor e I2P. grazie ad essi, il terrorismo islamico è riuscito ad espandersi oltre il Medioriente e a trovare il modo di finanziare le proprie attività attraverso canali illeciti paralleli che seguono tre passaggi fondamentali:

1. Raccolta

2. Occultamento del flusso

3. Impiego del provento illecito

Tali transazioni provengono da simpatizzanti, affiliati e sostenitori Isis, da traffici illeciti di droga (captgagon, utilizzato per stordire e non esitare sul campo di battaglia) e dalle esportazioni di petrolio. Oltre al sostentamento finanziario, il web occupa un posto di rilievo soprattutto per quanto riguarda la propaganda ed il reclutamento. Fare propaganda online vuol dire aprire una serie di piattaforme volte alla diffusione di immagini, video, messaggi jihadisti, manuali di addestramento e messaggi subliminali. Dalla morte del leader al Baghdadi, l'uso della propaganda online si è fatta più intensa approfittando del Lack of knowledge e applicando una strategia comunicativa:

- Telegram come social principale

- You tube

- App: Facebook, Instagram, Ask.fm, Whatsapp, Viber, Tumblr, Twitter.

Ogni canale è un punto di contatto per i reclutatori, i quali spesso sono appartenenti ad una sfera clericale. Al Battar è uno dei più vecchi sostenitori dello stato islamico in campo mediatico e munito di traduzioni in inglese. Nel 2018 subisce un depotenziamento dovuto al collasso del califfato in Siria e Iraq. Il 2019 è stato il continuo ristabilirsi della forza mediatica jihadista al fine di migliorare le scarse finanze dovute alla poca attività. Risollevarsi è stato possibile in parte grazie ai combattenti europei, reclutati tramite il web, tuttavia non è bastato alla ripresa totale, per questo, c'è stata la definitiva caduta di siti come:

- Akhbar al-Muslimeen;

- Haqq;

- Sawarim;

- Al Ghuruba.

L'aiuto fornito dai foreign fighters è la traduzione dei messaggi jihadisti dall'arabo alla propria lingua, permettendone la divulgazione oltre confine. L'apparecchiatura di alto livello ha mostrato al nemico di non essere meno equipaggiato di lui, pur mantenendo la gerarchia nel sistema di controllo. Il Central Media Office Isis è sede centrale delle attività di propaganda video online, seguono:

- Al Hayat

- Al Furqam

- Amaq Agency

- Al Abd Al Faqir

- Muhajir Al Ansar

- Al Yaqin

- Al Ajnad

- Remah

- Al Wafa

- Al Dhakhair

- Furat media foundation

- Al Sunny

- Turjuman Al Asawirti

- Muntasir media

Canali provenienti da tutto il mondo ed aventi un proprio logo, riconoscibile in alto a destra di ogni video di loro provenienza, attraverso la lettura del logo si può dedurre da che parte del mondo provengano. Elemento caratteristico unitario è l'introduzione ai filmati con l'inno Isis e la bandiera nera. 
Le riviste proposte dall'Isis sono le seguenti:

- Al Naba (nuovi argomenti) è la rivista più importante scritta in lingua araba. Tratta delle vittorie e dei successi dell'organizzazione e istiga i sostenitori a portare avanti gli obiettivi.

- Ashabah Al-Khilafah (giovinezza del califfato): non è una rivista ufficiale ma incoraggia aprenderne parte. Il target di riferimento sono i giovani musulmani. Agisce sotto sorveglianzadi Abd Al-Faqir.

- From Dabiq to Rome: rivista online non ufficiale in lingua inglese.

- Al Anfal (rovine di guerra): nasce nel tardo 2017 e pubblica ogni 10 giorni. Prodotto da Aldar' Al-Sunni foundation. I contenuti delle sue pubblicazioni istigano i lupi solitari a prendere coraggio e ad agire. Inattivo negli ultimi anni.

Ognuna di queste riviste opera sotto il controllo delle rispettive province di appartenenza, le principali sono Iraq e Siria ma si propagano fino in estremo oriente. La persuasione su internet è facilitata dall'uso dell'anonimato, questo rende più semplice il lavoro del manipolatore. Da anni ormai, i terroristi investono sulla manipolazione psicologica dell'individuo, il quale si ritrova coinvolto in un ciclo suddiviso in cinque fasi. Il Professor Anthony Stahelsky, in uno studio condotto presso la Central Washington University ha individuato cinque passaggi di condizionamento psicologico virtuale utilizzato dai gruppi jihadisti:

1. Depluralization

L'isolamento dell'individuo sta alla base del sistema. La vittima è spesso una persona che presenta complessità a livello psichico e sociale ed ha difficoltà ad inserirsi in società per via di sentimenti di rifiuto verso di essa. La causa che innesca nei soggetti di riferimento questo tipo di risentimento è la mancanza di una figura paterna nell'età infantile $(0,5$ anni) fase in cui il bambino apprende dalle circostanze favorevoli o sfavorevoli che andranno ad influenzare il suo modo d'essere per tutta la sua vita e avviando la formazione del super io. Si lascia l'amorale del fanciullo per andare in contro all'autorità dei genitori, che si manifesta con atti o concessioni d'amore o castighi a scopo educativo. Quindi l'innescamento di un'amorale insegna al bambino l'amore e cosa può comportarne la perdita. I soggetti che non hanno beneficiato di una figura paterna sono più propensi a cercarla al di fuori, spesso inciampando nella malavita. Il terrorista che vuole "iniziare" la sua vittima deve avere abbastanza informazioni riguardo al suo passato per capire come agire. La mente dei "Senza padre/ senza famiglia" è più malleabile ed introducibile ad una società che finge di comprendere i suoi sentimenti di abbandono, degli individui che hanno avuto gli stessi disagi e le stesse mancanze. Tutto ciò accade all'interno di communities virtuali, blog, forum, social media. Per il persuasore è più semplice individuare nuovi militanti grazie alla profilazione che avviene sui social, si tratta quindi di prendere in considerazione la maggior parte di informazioni rilasciate su internet e comparare ai modelli previsti.

2. Self-deindividuation

Annullamento, azzeramento dell'identità dell'individuo, pertanto, si passa all'azzeramento dell'uomo come persona. In breve, si tenta di dare priorità e valorizzare ciò per cui il soggetto è più predisposto a fare, esaltando quegli aspetti ed apprezzandoli a discapito di un passato sofferente e privo di riconoscimenti. L'indottrinamento non è altro che un percorso formativo dell'aspirante estremista durante il quale viene istruito partendo dalle attività base quotidiane e all'osservazione di regole comportamentali da svolgersi in perenne anonimato. L'iter dell'apprendista varia da pochi mesi ad anni di addestramento e si compone di materiale mediatico comprendenti guide ed istruzioni in formato digitale.

\section{Other deindividuation}

Il soggetto avrà acquisito un proprio modus pensandi ingannato dalle attenzioni del manipolatore. Tutto ciò che differisce da esso assume i connotati dell'ingiusto e del demoniaco, per questo, in questa fase è essenziale estremizzare ed accentuare il sentimento d'odio verso la società e verso tutti coloro che non fanno parte del suo percorso religioso. A questo accrescere d'odio equivale un'alterazione della realtà, ogni emozione legata al risentimento emerge nel soggetto e trova asilo nei suoi pensieri e nelle sue azioni.

\section{Dehumanization}

In questa fase, tutto ciò che è differente dall'idea religiosa islamista e considerato nemico ed infedele, un meccanismo diabolico che porta alla distruzione di quegli ideali considerati giusti e doverosi nei confronti della società islamica. L'occidente, in quest'ottica merita l'eliminazione, una perdita totale di sentimento umano, come ci suggerisce il nome stesso della fase, mirata alla perdita concreta di vite umane in onore di un Dio che predica la morte.

5. Demonization

La demonizzazione rappresenta il processo conclusivo del ciclo di reclutamento ed addestramento in cui si demonizza il nemico. Non è altro che un processo in cui si posiziona l'uomo occidentale ed infedele al pari di un demone distruttivo. La mancanza di risentimento verso le proprie azioni, favorito dalla perdita dell'umanità permette ai nuovi terroristi di adattarsi agli orrendi misfatti a cui spesso abbiamo assistito.

I passaggi qui descritti sono assolutamente simili nei loro contenuti alle fasi individuate dal Professor Mastronardi V. circa il reclutamento adottato dalle sette sataniche. In questo caso ci sono quattro fasi:

1) Reclutamento: con tecniche di avvicinamento e tecniche di vendita, quindi adottando test di personalità, libri, conferenze e corsi, in questo modo è più semplice capire chi è predisposto alla manipolazione mentale e chi no.

2) Isolamento: perdita di punti di riferimento materiali e affettivi in modo da allontanare l'individuo dalla realtà in cui si trova. Sottoporlo a love bombing, un estremo uso di gentilezze ed accorgimenti che inducano il prescelto ad identificarsi nella nuova società che saprà amarlo più di quanto abbia ricevuto fino al momento dell'entrata. Assenza di privacy e controllo dei movimenti, partecipare economicamente alla crescita del gruppo. Deresponsabilizzazione.

3) Indottrinamento: incoraggiamento all'obbedienza, Cambio nel vestiario e nei comportamenti, persuasione del soggetto rendendolo protagonista e prescelto per una missione divina. Uso di preghiere e formule critiche. Dieta imposta con aumento di zuccheri e poche proteine, ipnosi, punizioni/premi per i risultati raggiunti, messaggi subliminali.

4) Mantenimento: sottoposizione ad intensa attività fisica e mentale, testare la sua resilienza con privazioni di varia natura, scoraggiare iniziative personali, pressione psicologica, nenie, ricatti, senso di paura per una conseguente punizione (divina o corporea), linguaggio criptico per depistare e rimanere anonimi.

In entrambi gli studi risiede un'abile manipolazione psicologica dovuta al completo isolamento del soggetto, allontanandolo da ogni tipo di influenza esterna, sottoporlo ad ipnosi se necessario e se possibile e alla love bombing, ossia, far credere all'individuo di aver trovato un nucleo di appartenenza, un gruppo in cui potersi rifugiare e trovare conforto, in realtà sarà lo stesso gruppo ad indurlo a deumanizzare e demonizzare il resto della società attraverso tappe di disimpegno morale, in cui si inseriscono: 
- Giustificazione morale

- Etichettamento eufemistico (finire=uccidere)

- Confronto vantaggioso

- Dislocazione di responsabilità

- Diffusione di responsabilità (spostare la colpa al gruppo)

- Umanizzazione del proprio aggressore

- Attribuzione di colpa (provocazione)

- Training disinibitorio progressivo (disagio e riprovazione vengono meno)

- Auto-inganno

(Mastronardi V. 2002)

Protagonista dell'evoluzione tecnologica è il combattente Inghimasi, un militante che al momento del reclutamento sceglie di utilizzare ogni arma e metodo a sua disposizione per provocare il maggior numero di vittime possibili, sacrificandosi se necessario. La sua origine risale al 1080 d.C, quando un gruppo di Naziri attaccò il Medioriente per far prevalere le proprie idee politiche e piegare il volere dell'altro. Essi mantennero il dominio per circa 300 anni. A riprenderne il concetto fu Al Qaeda, divulgandone il significato nel 2011 per indicare i propri soldati impegnati sul fronte iracheno. Al principio, l'inghimasi sceglieva di agire munito di armi leggere e di una cintura esplosiva, seguendo il significato originario della parola Iinghamasa: essere immerso, tuffarsi, impegnarsi completamente in una missione di non ritorno se le circostanze lo richiedono. Il combattente kamikaze differisce per il fatto di non avere scelta sul campo, il suo compito è quello di farsi esplodere. Si riassumono qui i caratteri salienti di entrambi i profili.

Combattente Kamikaze (Shahid)

- Età 20-30 anni

- Musulmano

- Combattente straniero figlio dell'immigrazione

- Consapevole di dover morire

- Pone se stesso prima della causa

- Si definisce martire

- Mercenario e abile con le armi

- Esperto in tattiche di guerra

Combattente Inghimasi (istishhadiun)

- Forte fede in Allah

- Addestramento Muqatil (combattente)

- Possibilità di scelta se ricorrere al suicidio

- Fa parte dell'élite di corpi speciali

- Si mimetizza col nemico

- Lupo solitario

- Apripista di altri militanti

- Ad esso sono destinate le più alte stanze del paradiso

- Agisce con armi leggere

- Può ritornare alla base a missione compiuta

- Importante rumore mediatico.

La percentuale di età che con più frequenza rimane attiva sul web è quella che comprende il range di età 15-19 anni, per questo, l'ultima frontiera della propaganda jihadista arriva su uno dei social di ultima generazione: TIKTOK. Esso si presenta come un social per la condivisione di brevi video accompagnati da un sottofondo musicale e filtri per l'immagine, appartiene alla compagnia cinese "ByteDance", debutta in Cina nel 2017 e registra circa 110 milioni di utenti negli Stati Uniti. Ora è disponibile in 150 mercati e 75 lingue, la fascia d'età più attiva è proprio quella dei minorenni. Alcuni account di jihadisti hanno avuto occasione di aggirare le policy antiterrorismo ed essere pubblicati, ma dal 2019, secondo quanto riporta il Wall Street Journal dai dati dello Storyful, circa una dozzina di account sono stati scoperti e bannati. Il portavoce del social media di stampo cinese si dice preoccupato per come facilmente si riesca a sviare i controlli più rigidi, per questo si sta dando la priorità alla sicurezza degli utenti e delle generazioni più a rischio, puntando sulla rapida individuazione di presenze inopportune. In Italia si è provveduto ad effettuare operazioni per crimini online della jihad. Lo spostamento dei messaggi, avvenuto tramite il canale Telegram (social crittografato) per arrivare alle piattaforme virtuali esplicate poc'anzi, lo studio della George Washington University registra circa 85000 contatti e canali bannati riconducibili al califfato e trasmessi prevalentemente in arabo. Sul territorio italiano c'è l'assenza di veri e propri centri jihadisti, perciò ci si mantiene ancora lontani dalla situazione nel resto d' Europa, tuttavia, il Vaticano resta uno degli obiettivi più ambiti dai terroristi islamici ed è riconducibile all' Hadit (racconto sulla vita del profeta Maometto), secondo cui dopo la presa di Costantinopoli sarà la volta di Roma. Le operazioni perpetrate in Italia contro le cellule jihadiste sul web riguardano i gruppi: Ghulibati a Rum (Gal) e Ansar al Khilafah fi Italia (Aki), con sede nel nord Italia (Milano, Torino e regione veneta), se ne forniscono quattro esempi:

- Operazione Simm (veleno): contro la diffusione di ricina ed antrace, sostanze altamente tossiche per l'uomo.

- Indagine Taliban: contro gli inneggianti al califfato islamico sui social utilizzando tattiche di dissimulazione

- Operazione Ansar: indagine contro un canale telegram (Khilafah News Italian) creato da un cittadino italiano di origine tunisina

- Operazione Lupi del Deserto: Shalabi Issam Elsayed Aboulemayem, indottrinato su internet, reclutatore e portavoce del messaggio jihadista sul web tramite l'agenzia Amaq e la rivista $\mathrm{Al} \mathrm{Naba.} \mathrm{Il} \mathrm{su} \mathrm{nome} \mathrm{è} \mathrm{usato} \mathrm{per} \mathrm{indicare} \mathrm{altri} \mathrm{militanti}$ con i suoi stessi doveri e caratteristiche:

- grande dote comunicativa

- natura ibrida

- reclutatore

- intranei alla macchina propagandistica Isis

- disponibilità nel partire

- attenzione all'anonimato

- predisposizione alla leadership.

\section{Le forme di comunicazione: modalità di interrogatorio e linguaggio non verbale con approfondimento verso i terroristi islamisti}

Al fine di recuperare indizi utili alla realizzazione del profilo criminologico è indispensabile l'approfondimento nell'ambito della comunicazione non verbale (CNV); per tale scopo ci viene in aiuto l'interrogatorio e le tecniche di intervista che esso concerne. L'interrogatorio è regolato dalla norma vigente nel Codice di procedura penale all'art. 64, concernente le Regole generali per l'interrogatorio. Esse non consentono l'uso di "Metodi o tecniche idonei ad influire sulla libertà di autodeterminazione o ad alterare la capacità di ricordare e di valutare i fatti" mantenendo intatta la dignità della persona. Le modalità di interrogatorio contemplate dalla legge sono di tre tipi:

1. Difensiva

2. Investigativa

3. Di garanzia

La tragedia dell' 11 settembre 2001 ha conferito un nuovo volto all'asse di interesse geopolitico. L' America diventa un bersaglio vulnerabile per la cellula di Al Qaeda e le forze di polizia e di intelligence hanno iniziato una cooperazione volta alla prevenzione adot- 
tando metodi coercitivi e spingendosi oltre il limite consentito dalla legge. Le tecniche di interrogatorio hanno causato 119 vittime jihadiste, sospettati di appartenere ad Al Qaeda senza ottenere alcuna informazione. La loro resilienza è dovuta al tipo di addestramento ricevuto nel Daesh. L'enhanced interrogation comprende:

- Insulti

- Nudità

- Posizioni stressanti

- Minacce ed umiliazioni

- Digiuno

- Insonnia

- Vessazione

- Bagni d'acqua ghiacciata

- Waterboarding

Quest'ultima trae origine dall'inquisizione spagnola del 1500 "Tormento de aqua o toca" con la quale si provoca nell'indagato una sensazione di annegamento ad intermittenza, aggravato con del cellophane sul volto per creare delle vere e proprie crisi isteriche. Dopo lo scandalo della tortura su Abu Grahib (torturato 83 volte con waterboarding) la CIA ottenne il dissenso del dipartimento di giustizia e le tecniche vennero sostituite con delle alternative, le quali rispecchiavano quelle di sopravvivenza contenute nel programma Sere addestramento riservato a piloti delle forze speciali, e prevedevano tre fasi:

1. Ottenimento dell'informazione

2. Convertire/rieducare

3. Piegare la volontà

Interrogare un sospettato, soprattutto se resistente, richiede professionalità e conoscenza della psico-dinamica. Al fine di evitare discorsi fuorvianti da parte dell'intervistato, l'interrogante deve riuscire ad incanalare il discorso verso eventi e fatti anziché sulle emozioni. Alcuni suggerimenti sono:

1. Il silenzio

2. I rinforzi verbali (ah! Bene!)

3. La ripetizione (sinonimo di comprensione)

4. Rinforzi selettivi: "Ti ha rovinato!"

5. La ricapitolazione: "Lei sostiene che passeggiava lungo il viale, giusto?"

6. La riflessione: "Quindi deduco che lei era fuori di sé"

7. Elaborazione: "Se era preso dall'ira come mai fu visto sorridere un attimo prima?"

8. Chiarificazione: "Vorrei conoscere meglio il suo pensiero"

9. Spiegazione. "Questa è solo la premessa"

10. Interpretazione in base al proprio punto di vista

(Scheanfield e Perlman, 1981)

Le interviste si dividono in: investigativa e cognitiva. la prima intervista si sviluppa in Inghilterra ed è utilizzabile in un approccio cognitivo e per avere una conversation management, e segue l'acronimo PEACE:

$\mathbf{P}$ preparation

E engage

A account

C close

E evaluate

Nell'intervista cognitive si richiede di ricreare e rielaborare i dati ed individuarne delle teorie: ricreare il contesto criminoso, riferire pensieri dell'intervistato ed eventi discordanti dalla originale versione, trasporre l'evento in terza persona. Gli esperti suggeriscono dei metodi funzionali: domande multiple, di tipo chiuso, utili a restringere il campo, e domande con schema ad imbuto o imbuto rovesciato: iniziando con schemi generali per arrivare al dettaglio e viceversa. Per valutare un dialogo bisogna tener conto di elementi quali:
- indicatori del timore: sudorazione, senso di prurito, pianto, agitazione, atteggiamento paralizzato

- indicatori di menzogna: sguardo rivolto in alto a sinistra, mano davanti alla bocca, toccarsi il naso, diminuzione dei gesti illustrativi e brevità delle risposte

Inoltre, si considerano episodi di falsi ricordi, simulazione e fattori come maltrattamenti e vessazioni per non cadere nell'errore di giudicare un sospettato solo in base alle conseguenze del suo stato di ansia.

Il manuale Kubark (1963), messo in atto dagli agenti della CIA contro i militanti di Al Qaeda è l'esempio di coercizione fisica e mentale basata su violenza, isolamento e regressione ipnotica. I metodi qui espletati, nel tempo potrebbero ledere in modo permanente a livello psicologico e per fa sì che ci sia comprensione e capacità di risposta alle questioni si tiene conto di un limite da non oltrepassare e non alterare le sue abilità. I passaggi presenti nel manuale sono:

1. arresto

2. detenzione

3. privazione di stimoli

4. minacce

5. indebolimento

6. dolore

7. alta suggestionabilità ed ipnosi

8. narcosi

Il protocollo attuato dall' FBI si basa, invece, su passaggi basati sull'affronto e confronto col soggetto esaminando lo status comportamentale. Joe Navarro, agente speciale dell'FBI ed esperto di comunicazione non verbale suggerisce di porre attenzione su quattro aree:

1. discomfort

2. enfasi

3. sincronia

4. gestione della manipolazione (in particolare in soggetti psicopatici e abili manipolatori)

(M. Strano, 2007)

Alcuni dei tranelli psicologici più famosi e di successo di uso in campo investigativo sono il: knowledge bluff, in cui chi interroga mostra l'impressione di sapere molto più di quanto in realtà si stia dicendo; il metodo fixed line-up, ossia indicare il sospettato come il colpevole o il metodo bluff on a split, in cui il prigioniero in attesa di essere interrogato viene fatto entrare e messo di fronte ad una presunta confessione scritta del suo complice. (Cairati, 1987)

Strumento di valutazione dei dati di un interrogaatorio è la L.D.I.G (Lie Detection Interrogation Grid). Con essa si possono analizzare ciò che viene registrato durante l'interrogatorio con tecniche audio e video. Prevede tre aree di osservazione.

1. Area di attenzione generale

2. Area di attenzione audio sulla linguistica e paralinguistica

3. Area di attenzione video

In applicazione delle precedenti teorie circa l'interrogatorio coercitivo si è preso in considerazione un episodio della serie televisiva Criminal Minds, fedelmente riprodotto su documentazione reale ed in collaborazione con il Federal Bureau of Investigation. Nel caso studio, la scena evidenzia il ruolo del profiler e la posizione dell'interrogato jihadista. Gli elementi di cui tiene conto l'interrogante sono:

- Linguaggio del corpo

- Addestramento del soggetto

- Capire le motivazioni dei suoi gesti

- Analizzare la scelta delle parole 
- Osservare il comportamento in fase di quiete e di ansia

- Spingere per ottenere informazioni

- Giocare d'astuzia

Nella sequenza di immagini studiate troviamo la figura del terrorista islamico inizialmente preda degli uomini dell'esercito, insultato, svestito e picchiato. Nonostante le continue offese subite, egli mostra una forte resistenza, è quasi indifferente alle torture e si rifugia nella preghiera e nel silenzio. Il profiler entra in gioco in modo completamente diverso, tentando di stabilire un contatto con l'interrogato, rivestendolo, inducendolo a parlare e prestandosi all'ascolto. Una volta catturata l'attenzione del prigioniero di instaura il dialogo. Durante la conversazione, il profiler studia i movimenti facciali, il colore della pelle ed i tratti linguistici che potrebbero ricondurre ad una determinata area geografica. Da non sottovalutare l'intelligenza del terrorista che, in ogni circostanza, se costretto a parlare tenterà di ingannare l'interrogante con ogni mezzo. Per evitare di farsi ingannare, l'elemento di spicco è L'atteggiamento dei discorsi che spesso non coincide con la linguistica del corpo: tensione, pugni chiusi, sopracciglia aggrottate, micro espressioni, infatti, come sostenuto da Desmond Morris, autore di numerosi volumi di sociologia umana, il miglior modo per ingannare il prossimo è limitare $\mathrm{i}$ segnali alle parole e alle espressioni del viso, poiché il resto del corpo, mentre è impegnato a mentire, resta fermo o si riduce a piccoli movimenti. Stessa teoria e procedura di analisi della menzogna è stata applicata per Abu Moussa, ex militante Isis, pentito al momento della cattura nella città di Raqqa. Il video che ha permesso un'analisi approfondita dei suoi movimenti è reperibile sul canale you tube Inside Over: gli occhi della guerra, in un reportage di Fausto Bisoslavo. Fin da subito il soggetto, di origine tunisina, risulta infastidito dalle riprese video, mente sulla reale occupazione all'interno dell'Isis, è diffidente, si muove spesso, ammicca. Il suo pentimento risulta rapidamente poco credibile, perciò l'ipotesi di menzogna può essere portata avanti e ulteriormente accreditata dalla volontà di volersi ricongiungere, senza complicazioni, alla sua famiglia, tenuta prigioniera in un campo profughi.

\section{Ricostruzione del profilo criminologico del soldato dell'Isis e la migrazione: affluente del terrorismo}

Elemento di particolare rilievo nella realizzazione del profilo criminologico è quello culturale e sociale. Negli ultimi anni si è assistito ad un calo degli attacchi in occidente, ma la sconfitta in Iraq e Siria potrebbe essere l'incipit per uno spostamento. Il metodo più utilizzato per espandersi altrove è la migrazione clandestina: rotta mediterranea passando per Libia e Tunisia oppure la rotta balcanica via terra, raggiungere luoghi come: Afghanistan, Kazakistan, Tagikistan, Turkmenistan, Kirghizistan, Bosnia e Russia. L'insediamento sarebbe favorito da situazioni decadenti all'interno dei paesi ospitanti, come per i conflitti o povertà, scarso controllo delle autorità, stesse dinamiche che portarono alla nascita dell'Isis in Iraq e Siria. Territori in cui vi era un alto tasso di disoccupazione ed analfabetismo, dovuto alla caduta del partito Baath nel 2003. L'organizzazione islamista si presentò come portatrice di reddito e assistenza, in realtà ciò che ne conseguì fu una rigida condizione di vita, resa primitiva, controllata da leggi opprimenti. Somalia, Nigeria e Colombia rientrano tra $i$ candidati più a rischio di radicalizzazione islamica. L' Italia, in proporzione ad un'analisi di rischio basata su immigrazione e insediamento di comunità islamiche presenta un rischio più basso al nord e al centro, mentre è crescente nel sud.

Un'analisi UNHCR del 2018 stabilisce che: Guinea, Siria,
Marocco, Mali, Afghanistan, Iraq e Tunisia sono tra i paesi inscrivibili alla migrazione passando per la rotta mediterranea. Dall' Africa centrale si compone il puzzle della criminalità: dia trafficanti di migranti, al contrabbando, ai traffici illegali, elementi che contribuiscono al business dell'illecito. L'aumento dei flussi è iniziato a partire dalle primavere arabe del 2011, una svolta politica e sociale di importazione occidentale alla quale i paesi dell'area mena non hanno saputo adattarsi a pieno. Il Niger rientra tra le zone di passaggio, in cui affluisce il più alto numero di migranti sub-sahariani. Si registrano stime che variano attorno ai 296000 immigrati provenienti dal Niger, dai 22 ai 25 anni con prevalenza di sesso femminile, per via del traffico della prostituzione. Il terrorismo derivato dai flussi migratori è stato rinominato "Insurrezionale" (Berlotti, 2015), in quanto si adatti alle nuove circostanze di profitto. Nel termine insurrezione ritroviamo quegli atti illegali e politicamente motivati volti a perseguire fini terroristici. Dal 2014, l'Isis in Europa ha raggiunto il suo picco solo nel 2017. In questo arco di tempo, di 895 attacchi terroristici riusciti e falliti, il 19\% riguarda radicalismi islamici, su un milione di migranti, la relazione con il terrorismo è pari allo $0,00002 \%$. Seppur in numero irrilevante, gli ultimi attacchi rivendicati dall' Isis sono stati effettuati da singoli (lone wolves) o da gruppi di poche persone, ad esempio l'attacco al Bataclan, in Francia, di cui otto partecipanti vi erano due immigrati. Su un campione dati pari al $62 \%$ del totale degli attentati (2015-2019):

$65 \%$ riconducibile ad immigrati di prima generazione, $33 \%$ regolari, 31,6\% seconda e terza generazione, $17 \%$ convertiti all'Islam. Il ruolo ricoperto dai foreign fighters, in un rapporto Ispi nel periodo 2014-2016, con un dato in crescita del $45 \%$ su 42 piani terroristici accertati, sembra destare preoccupazione in quanto verta verso il rischio "Effetto Blowback", ossia agire nel paese di destinazione legittimando le azioni in nome del califfato. Un ritaglio è destinato al caso del Belgio. Il paese in analisi si presenta come un ventaglio di varie nazionalità, fenomeno riconducibile ad una ventata di immigrazione dal 2000 in poi. Il Belgio è suddiviso in tre grandi etnie: $54 \%$ fiamminghi, $37 \%$ valloni, $0,7 \%$ tedeschi. Tra le minoranze ritroviamo:

- Italiana $1,5 \%$

- Francese $1,3 \%$

- Olandese $1,2 \%$

- Turca e marocchina $0,8 \%$

- In percentuali minime polacchi, romeni e spagnoli.

La sua struttura si basa su una "Pilastrizzazione" della società dovuta alla frammentaria presenza culturale. Nel corso degli anni si sono costruite barriere linguistiche incentivando il separatismo. Il sistema federale è a doppio livello, distribuito in parti eguali al sistema centrale e alle singole regioni. A livello religioso troviamo una tripartizione tra calvinisti, cattolici e laici, mentre la restante minoranza professa l' Islam, seppur in minor numero, essi hanno costituito una piccola comunità chiusa "Little Marocco", nel quale, grazie all'infiltrazione del giornalista Hindi Fraihi, specializzato in cultura araba, si è scoperto il metodo di indottrinamento della jihad: predicano per un sistema più rigido, che imponga alle donne 1 'uso del burqa, e l'avvio dell' indottrinamento/ manipolazione dei più giovani. Le vittime più influenzabili sono i ragazzi di strada, senza un nucleo di appartenenza. La jihad ha trovato rifugio in Belgio, in quanto vi risieda più grande sede in Europa della comunità musulmana (Cicb). Inoltre, la stazione di Bruxelles Gare $d u$ midi ospita il più grande mercato domenicale d' Europa ed è il punto di passaggio del mercato nero che distribuisce armi ai terroristi. La radicalizzazione in Belgio comincia nel 2011, a seguito di un malcontento generale circa la situazione multietnica e di segnali di sofferenza lanciati dalla vicina città di Anversa, muovendo i primi passi per la nascita di Sharia4Belgium (Sharia for Belgium) 
un gruppo di radicali il cui interesse unico era la diffusione della jihad e la guerra dell'Islam. Fouad Belkacem, leader dell'associazione criminale, di origine marocchina ha cominciato la divulgazione del messaggio jihadista con propagande di stampo salafita. Raccogliendo un gran numero di persone, molti di essi hanno scelto di partire per il fronte siriano nel 2011, per poi tornare in patria e portare gli insegnamenti della guerra. Belkacem venne incarcerato un anno dopo e sostituito da un suo amico Jean Louis Denis, anch'egli arrestato poco dopo con conseguente crollo dell'organizzazione.

A questo punto si è passati alla raccolta delle informazioni per procedere con il lavoro di profiling. Innanzitutto, per costruire un profilo si parte da sei interrogativi anglosassoni: who, what, when, where, why, how. Tuttavia, il lavoro del profiler non può essere svolto per ogni crimine ma solo per quelli che racchiudano alcuni valori emergenziali:

- Crimini inspiegabili

- Comportamenti sadici

- Assenza di motivazione

- Omicidi, sventramenti, torture, amputazioni, occultamenti, azioni seriali

- Lettere terroristiche

(Mastronardi V., 2005)

Prima di conoscere il responso delle varie associazioni di informazioni, si è voluto presentare il modello di profilo qaidista per trarne degli elementi di confronto.

Origine: araba

Fede: musulmano conservatore

Formazione/addestramento: sul campo

Attentatori: kamikaze (shahid), combattente semplice (Mujahideen)

Eta media: 34 anni

Tasso di istruzione: medio-basso

Livello economico: basso

Requisiti psico-fisici:

- Astuzia

- Intelligenza

- Calma

- Resistenza

- Freddezza

- Divieto di bere alcolici o avere rapporti sessuali

- Può comportarsi come un occidentale solo se in missione e sotto copertura

(Mastronardi V, Leo S., 2005)

Oggetto comune degli appartenenti alla cellula di Al Qaeda è la assoluta fedeltà all'interpretazione rigida ed estrema del Corano oltre che all'ex leader Osama Bin Laden. La devozione ai principi islamisti ha fatto in modo di sviluppare una gerarchia all'interno dell'organizzazione con al vertice il leader carismatico e capo della Shura Maijils, consiglio di supervisione composto da: commissione religiosa, commissione economica, commissione militare, commissione comunicazioni. Ognuna di queste commissioni comprende al loro interno degli esperti o tecnici specializzati e che si preoccupano di inviare il messaggio jihadista in tutto il mondo ed avere degli appoggi in piccoli gruppi a livello internazionale. Obiettivo mai pienamente raggiunto, in quanto i soldati trovavano la loro forza e motivazione nel capo Bin Laden, venute meno alla sua morte. Un'evoluzione differente spetta allo Stato Islamico. Il profilo si avvale di una panoramica dei paesi di provenienza dei migranti e l'influenza che danno al paese di destinazione, ricoprendo spesso, il ruolo carismatico che porta le seconde generazioni a seguire le orme dello Stato islamico, creando il fenomeno degli Homegrown (soggetti radicalizzati all'interno del proprio paese, con un passato criminale e senza esperienza all'estero). Secondo Claudio Bertolotti, direttore dell'osservatorio sul contrasto al terrorismo nel rapporto sul radicalismo si possono individuare dei macro gruppi in cui racchiudere le principali etnie dei terroristi jihadisti e delle loro famiglie. Il paese più rappresentativo è il Marocco con una percentuale del $21 \%$ e Algeria con il 10\%, questo dato influisce sul paese più colpito dagli attacchi che è la Francia seguita dal vicino Belgio, in cui gli attacchi da terroristi provenienti dalle due regioni sale al 31\% del totale. La Spagna subisce l'affluenza di marocchini ed in particolare di donne seguaci dello stato islamico, le quali ricoprono il $35 \%$ degli arresti nello stato. Nonostante le percentuali inferiori, Iraq, Siria e Libia rappresentano il quadrante dei foreign fighters, portatori del messaggio jihadista in paesi europei, aumentando, nel tempo, sia la qualità che la quantità degli attacchi subiti. Il target riconosciuto come preponderante negli attacchi in Europa (2014-2019) ha compreso l'operatività di 162 soggetti, l'età mediana è di 26 anni in una scala di interesse che va decrescendo di circa 5 anni ogni anno, andando così dai 34 anni del 2014, (20\%del totale) ai 22 anni del 2017. C'è quindi una sorta di schema verticistico che vede all'apice gli uomini che si avvicinano alla mezza età, e che spesso provengono da zone di guerra, fiancheggiati dai meno esperti non meno informati, e le nuove reclute, figli di immigrati che si affacciano alla cultura jihadista. Il terrorista del califfato islamico si presenta in molte varianti, per questo motivo numerosi studi hanno dichiarato impossibile costruire un profilo individuale unico. Riassumendo tutte le caratteristiche prese in considerazione nei vari ambiti qui trattati, ciò che ne scaturisce è riportato nei seguenti dettagli.

\section{Caratteri principali del soldato Isis contemporaneo}

- Età mediana: 26 anni in un range compreso tra 34 e 22 anni

- Origine/provenienza: Marocco, Algeria, Afghanistan, Tunisia, Siria, Francia, Senegal

- Livello socio-economico: medio-basso

- Livello di istruzione: medio-basso ( $87,7 \%$ del totale)

- Precedenti penali (44\% del contingente)

- Area di indottrinamento: web 55\%, carcere $24 \%$

- Ruolo nell'organizzazione: seguendo la struttura piramidale si hanno: leader, reclutatori informatici, foreign fighters, kamikaze, inghimasi, combattente semplice, lone wolves.

- Fede/ideologia: Islam radicale

\section{Caratteristiche psico-attitudinali del soldato Isis contemporaneo}

- Pericolosità: alta anche se in numero di partecipanti ridotto

- Recidiva criminale: non recuperabile se nato e cresciuto al fronte, parzialmente o del tutto recuperabile se nato e cresciuto all'estero

- Capacità cognitiva: non vi sono casi di infermità mentale

- Emotività: assente

- Predisposizione all'acting out: alta

- Capacità di mentire: affinata negli anni con l'aiuto della tecnologia

- Disturbi psichici: gli esperti non confermano disturbi psichici al $99,2 \%$

Gli elementi che sono stati presi in considerazione rivelano un'alta pericolosità, stante nel fatto che anche l'attacco di un singolo può provocare la morte o il ferimento di centinaia di civili, un esem- 
pio può essere un attacco di un kamikaze in una piazza affollata. Ogni soldato dello Stato Islamico proviene da una storia diversa, in particolare i combattenti stranieri, i quali sono appartenenti ad una cultura totalmente diversa da quella di un individuo nato e cresciuto nei luoghi del fronte. Questo determina non solo il loro approccio all'Islam e alla jihad ma soprattutto l'essere recidivi e nel caso, la possibilità di recuperarli attraverso programmi di rieducazione. Gli arruolati vengono selezionati previo test psicologico, per cui, un soggetto incapace di intendere e di volere non è predisposto alla guerra di Allah, in quanto si ricerchi astuzia e capacità cognitiva. Essenziale è la capacità di deumanizzare il nemico, porre ai minimi termini i livelli di emotività, aumentando di conseguenza il livello di pericolosità di un attacco e dell'acting-out criminale. Come precedentemente dimostrato, la tecnologia ed il web hanno incrementato l'abilità manipolatoria dei terroristi, affinando l'arte della menzogna. Stando al continuo calo dell'età registrato, dovuto soprattutto alla percentuale di giovani attivi su internet dai 19 ai 24 anni (81\%), l'attenzione si sposta automaticamente alle seconde e terze generazioni in quanto le più influenzabili, pertanto si ritiene necessario agire nei confronti dei più inesperti in forma preventiva ed arginare la nascita di fenomeni di terrorismo Indotto.

\section{L'approccio europeo ed italiano come formula risolutrice nella prevenzione al terrorismo islamico}

Ciò che contraddistingue l'Italia da altri paesi d'Europa in fatto di terrorismo islamico è l'assenza di attacchi massicci e di attentati a simboli del paese. Spesso si predilige l'indottrinamento online, in quanto sia più facile e veloce la ricerca di materiale di interesse. Stabilire un contatto diretto con enti quali Isis o Al Qaeda e decidere di attaccare a livello transazionale è complicato ma non impossibile, tuttavia, proprio per la difficoltà nella connessione tra le parti, si preferisce restare sul suolo italiano e preparare attacchi singoli tralasciando il collettivo. Paesi come Francia, Belgio, Inghilterra e Spagna sono stati il teatro d'azione del terrorismo islamico recente, l'Italia rientra nelle intenzioni di attacco jihadista, spesso rappresenta l'ultimo passaggio della loro conquista per il dominio mondiale e più volte, il Vaticano, simbolo della cristianità è stato oggetto di minaccia da parte dei terroristi. Per via dell'impossibilità di fare previsioni certe a lungo termine, il contrasto migliore rimane la prevenzione, a tal proposito si promuovono organizzazioni CVE (Coutering Violent Extremism). L'organizzazione si muove all'interno del progetto Mates, programma di prevenzione alla radicalizzazione, facendo leva sul Soft Power: coinvolgimento, principio di salvaguardia, territorialità, trasparenza. Essi muovono su tre livelli:

1. Macro-livello: contro-narrativa e narrativa alternativa in contrasto col messaggio jihadista.

2. Meso-livello: ingaggio positivo, ossia il confronto e l'informazione dei giovani sul tema del terrorismo con autorità e forze dell'ordine.

3. Micro-livello: attiva in casi singoli su sospetto o accertato jihadista.

In Italia, tra i profili riconosciuti, si passa dal profilo del laureato, a quello del criminale, dal tossicodipendente all'imprenditore di successo, non ci sono grandi comunità di musulmani, quartieri ghetto o flussi migratori riconducibili al colonialismo, come invece si registra nell'Europa del nord. Fino al 2016 sono stati 34 gli arresti, 57 le espulsioni e 110 i recuperati. L'analisi dei dati ci permette di dire che il nostro paese sta vivendo la fase embrionale che altri hanno già avuto, colpa dei continui flussi di migranti clandestini provenienti da rotte mediterranee e balcaniche e delle situa- zioni dei paesi nord-africani, e ricordiamo inoltre, che è stata la nascita in Siria e Iraq dell'Isis che ha trasportato le terze generazioni ad allearsi col l'esercito Daesh. Il problema delle carceri risiede nel fatto che i piccoli gruppi jihadisti che si formano all'interno sono isolati tutti insieme e in carceri di massima sicurezza e dividerli rappresenterebbe un ulteriore dilemma, i quanto potrebbero singolarmente indottrinare altri prigionieri criminali comuni, perciò il Dipartimento dell'amministrazione penitenziaria (DAP) ha trovato soluzioni tra dispersione e concentrazione. Differente è invece il problema di internet, che dal 2009 con l'inchiesta Niriyaha portato alla luce dei gruppi di convertiti ed ha visto la grande diffusione di materiale jihadista. A farne parte sono i giovani dai 18 ai 24 anni, coloro che sono più inclini alla globalizzazione. Le "lezioni" tenute sul web dai reclutatori possono arrivare a durare delle ore e sono tenute da uomini e donne. La partecipazione è attiva da ambo i sessi con una netta maggioranza degli uomini. A livello europeo la risposta è stata vasta e si è cominciato ad applicare le proprie dottrine a seconda delle necessità e dei contesti socio-culturali considerando: politica, sociologia, legislazione. L'iter seguito dai programmi CVE segue: macro-livello, mesolivello e micro-livello, attraverso di essi si vanno ad includere alle misure messe in atto dalle forze poliziesche un aiuto completo da tutta la società e quindi multidisciplinare che impegna il singolo cittadino a sentirsi parte di questa lotta, non per perseguire penalmente gli altrimenti non punibili, ma iniziare un recupero.

1. MACRO LIVELLO, contro-narrativa e narrativa alternativa: si tratta del canale comunicativo con cui i terroristi hanno tradotto le leggi domanda-offerta del mercato nel proprio business, alimentando il fascino della loro offerta con risposte semplici, appetibili. In risposta alla tecnica jihadista si consiglia di ricambiare con un altrettanto allettante piano comunicativo e diminuire l'appeal jihadista. Per contro-narrativa si intende il messaggio e le argomentazioni più indicate a contrastare la propaganda jihadista; la narrativa alternativa, invece, muove dubbi ed incertezze su quanto diffuso dagli estremismi, attaccando l'ipocrisia dei leader nell'interpretare il corano. Il ventaglio di opzioni persegue approcci positivi screditando la strategia e corruzione, tipica dell'estremismo radicale. Ulteriori strumenti necessari nella diffusione di un messaggio è la legittimità di chi lo diffonde, in questo caso sarà un Musulmano a tenere le redini, e sarà altresì un Musulmano ad attivarsi contro la jihad. Per ciò che riguarda il modo di diffusione è importante tener conto della qualità, in tema di piattaforme virtuali, pertanto il contrasto deve apparire non da meno, se non migliore, in modo da mostrarsi come la scelta più efficace. Londra ha ispirato l'Europa adottando la sezione Google "Redirect Method", utilizzata per ricondurre a chi digita parole chiave propense all'estremismo. Lo Stato mantiene il ruolo chiave nella lotta alla radicalizzazione, accompagnato dai mezzi di informazione, portavoce di messaggistica d'impatto visiva e auditiva, così come la sinergia tra entità statuali, collegamenti, partenariati, il tutto incentivato non solo dall'utilità del progetto ma da fondi nazionali ed europei. come sappiamo, in un mondo che si evolve e si avvicina per effetto della globalizzazione mediatica, musica e spettacolo contribuirebbero come elementi chiave entrando così nel quotidiano di tutti in un modo molto semplice: la televisione e la radio.

2. MESO-LIVELLO, contatto positivo: è il livello intermedio in cui si tenta di ampliare il più possibile la propaganda antiestremista tra i giovani. In questo caso si assume un atteggiamento più cauto avvicinandosi ai soggetti "a rischio" comunicando con loro, ponendo loro delle scelte e integrandoli in decisioni che riguardano la collettività. Partire dall'informazione nelle scuole è prezioso, in modo da poter creare una con- 
sapevolezza fin dalla tenera età ed aiutare il bambino/adolescente a saper prendere una scelta guidata e ragionata. Ammessi i valori di democrazia, senso civico, tolleranza e corretta interpretazione dei testi islamici. Il progetto RAN (Radicalisation Awareness Network) attivo dal 2011 collega tutti gli Stati europei per essere sempre connessi con team di esperti, forze dell'ordine, insegnanti, operatori sociali in ambito di terrorismo, ed ha coinvolto già più di 2000 lavoratori in vari settori:

- Comunicazione e narrativa (C\&N)

- Educazione (EDU) Partenze (EXIT)

- Giovani, famiglie e comunità (RAN YF\&C)

- Autorità locali (LOCAL)

- Carcere e libertà vigilata $(\mathrm{P} \& \mathrm{P})$

- Polizia e forze dell'ordine (POL)

- In ricordo delle vittime di terrorismo (RVY)

- $\quad$ Salute e sicurezza pubblica (H\&SC)

In Italia il progetto RAN ha preso piede a Torino, Lombardia e Friuli-Venezia-Giulia con le azioni di "Counternarrative for counterterrorism”, corsi di formazione per docenti sull'educazione alle differenze nella lotta al terrorismo, sistemi di preallarme carcerari e progetti locali.

3. MICRO-LIVELLO, interventi individualizzati ad occuparsi del micro-settore sono i CNR, Centro Nazionale sulla Radicalizzazione, ed i CCR, Centro di Coordinamento sulla Radicalizzazione, che rispondono alla prefettura di ogni regione. Le squadre sono formate da operatori sociali, psicologi, poliziotti, ex militanti nell'interesse delle strategie CVE. Il CRR svolge il compito di partire dal piccolo per arrivare a fare ciò che è richiesto nel meso e nel macro-livello, quindi occuparsi di prevenzione nei vicoli della società controllando le segnalazioni:

1. Dismissione delle segnalazioni: accertarsi che non siano false segnalazioni

2. Segnalazione alle forze dell'ordine

3. Predisporre un intervento: il CRR interviene con riabilitazione/de-radicalizzazione accompagnando l'individuo in una rieducazione del pensiero.

In merito al fenomeno della radicalizzazione e de-radicalizzazione, il Professor Vincenzo M. Mastronardi ha suggerito un metodo di approccio nel progetto Mates partendo da un'analisi storico individuale del periodo di riferimento e agire su un punto primario, secondario e terziario:

prima del processo di radicalizzazione

dopo l'inizio del processo di radicalizzazione

soggetti recidivi (importante tener presente la recuperabilità di un individuo radicalizzato): impegnativi/irrecuperabili; soggetti recuperabili con un piano individuale; soggetti potenzialmente recuperabili

Il campo d'azione si concentra sui tre livelli menzionati nel paragrafo precedente, e di cui si vuole fornire un approfondimento pratico:

Nel macro-livello (contro-narrativa/narrativa alternativa) lo studio suggerisce otto punti da seguire per aumentare l'efficacia del metodo:

1. Diffondere immagini favorevoli alle proprie forze

2. Ampliare gli effetti delle operazioni militari sul nemico

3. Condizionare gli atteggiamenti psicologici e atteggiamenti del nemico

4. Aggirare le censure, l'analfabetismo e gli ostacoli col nemico

5. Sfruttare le differenze etniche

6. Offrire spazio a tipologie belliche non convenzionali

7. Coadiuvare con operazioni umanitarie di peace keeping

8. Confezionare proiezioni ad hoc con casi ufficialmente dichia- rati e di cui si dispone di abbastanza materiale per poterne estrarre delle soluzioni.

Nel meso-livello (Ingaggio positivo) si sperimenta un approccio mirato a gruppi vulnerabili e predisposti al pericolo come le scuole.

$\mathrm{Nel} \mathrm{micro-livello} \mathrm{(livello} \mathrm{individuale)} \mathrm{si} \mathrm{passa} \mathrm{al} \mathrm{programma} \mathrm{di}$ de-radicalizzazione a seconda del grado di recuperabilità del singolo.

Il risultato è quindi un miglioramento/creazione dell'autostima, agire con una terapia psicologica e farmacologica in caso di psicopatologia; fare attenzione alle tossicodipendenze ed il graduale reinserimento sociale. Per fare ciò, occorre tenere presenti tutti gli aspetti di vita e le problematiche che affliggono l'individuo prescelto accompagnato da un buon grado di empatia nel comprendere le reali motivazioni.

Considerando l'aspetto psicologico del radicalizzato, bisogna tener presente il Brainwashing, lavaggio del cervello che può essere applicato sulle vittime in diverse modalità: violenze fisiche e psicologiche; istigazione alla violenza, che viene vista come l'unico modo per imporsi sull'altro; reagire alle critiche subite; si giustifica ciò che accade con la volontà di Allah (Inshallah); ritenere l'intolleranza sintomo di superiorità.

Conseguenze di un lavaggio del cervello legate all'insorgenza di psico-patologie latenti sono:

- Schizoide/paranoide

- Psicosi delirante

- Narcisismo accompagnato da disturbo antisociale, istrionico, dipendente, ossessivo/compulsivo. L'intervistatore deve giungere a capire il "perché" delle sue azioni e delle sue convinzioni e per farlo segue la scaletta fornita da Henry Tuck e Tanya Silvermam:

1. Ricerca dell'identità

2. Ricerca dell'identità sociale

3. Necessità di esprimersi con gesti eroici e missionari

4. Impegno e voto al credo

5. Comportamenti violenti

6. Necessità di avere un leader su cui fare affidamento.

Da tener presenti umiliazioni e difficoltà nel vissuto, sia in ambito familiare che in altri tipi di relazioni. Prioritari sono gli insuccessi e lo status sociale oltre che al grado di istruzione ricevuta, che può o meno influire sulle scelte dell'individuo. Gli errori che l'intervistatore deve evitare son riportati nelle tecniche e diagnosi di personalità di Passi-Tognazzi, (1964):

1. Porre domande che precludono una risposta aperta

2. Porre domande suggestive che possano suggerire la risposta

3. Chiede esplicitamente il perché

4. Prendere appunti

5. Restare impassibili

6. Dargli l'impressione di essere un caso da studiare, ma mostrarsi interessati alla sua storia, alle vicende che lo hanno spinto a percorrere determinati sentieri. È necessario, inoltre, congiungere ogni aspetto narrativo alla comunicazione neuro linguistica, con argomenti reali di fatti concreti e argomenti che riguardano le preferenze del soggetto, quali: valori, gerarchie e luoghi preferiti. L'essere persuasivi vuol dire creare l'intesa perfetta col soggetto intervistato. Nel dialogo con un jihadista è importante, inoltre, tener presenti tutti gli elementi caratteristici del credo jihadista, dall'etimologia della parola (jihad=sforzo) ai modi in cui il Corano indica il combattere per la jihad:

- Con il cuore purificandosi dal peccato

- Con la lingua predicando la giustizia

- Con la mano per punire i peccatori

- Con la spada contro gli infedeli.

Le correnti dell'islam da cui trarre insegnamenti sono: sunniti e sciiti seguaci della discendenza di Alì, entrambi condividono i 5 
pilastri dell'islam e l'essere Musulmano, quindi sottomessi ad Allah seguendo il Corano, cioè l'interpretazione, che insieme alle Hedith, (raccolta di leggi nella Sunnah) formano la Shari'a. Dal momento in cui un militante viene reclutato sceglie una vita da seguire che all'apparenza può sembrare la giusta causa:

- Innamorati della guerra

- Innamorati del martirio

- Innamorati della giustizia.

Per legittimare ogni sforzo ed ogni azione violenta contro il nemico ormai demonizzato si adoperano delle giustificazioni ascrivibili in 3 gruppi:

1. Giustificazioni morali, etichettamento eufemistico (scelta accurata delle parole per non dare l'evidenza di un atto violento) dichiarandosi altruisti in ciò che fanno.

2. Dislocamento di responsabilità (Allah dice questo), distorsione delle conseguenze (minimizzare il misfatto).

3. Deumanizzazione (ricatalogare ciò che è umano è ciò che non lo è), attribuzione di colpa (agire per difesa).

Osservando il modus operandi di tutti i profili legati all' Isis possono classificare i seguenti ruoli nell'organizzazione:

Foreign fighters: i loro profili sono tutti diversi, appartengono a culture diverse e di conseguenza ciò che li spinge ad arruolarsi non si riduce mai ad un'unica motivazione. Costoro partono dai propri paesi d'origine per combattere al fronte, spesso sono giovani, abili con la tecnologia, non interessati al credo islamico né all'inserirsi in una comunità islamica, cercano piuttosto di identificarsi in un gruppo. A facilitare la loro sottomissione al califfato è un particolare tipo di droga: Captagon, l'unione del Biocapton e Fitton che permettono loro di sopportare dolori e digiuni anestetizzandoli.

Kamikaze: età compresa fra i 20 e 30 anni, europei di seconda o terza generazione, sanno resistere a qualsiasi tipo di pressione fisica e psicologica e sono impassibili di fronte ad eventi come: prigionia, visione di massacri e uccisioni, sono calmi e intelligenti ma privi di empatia. Sacrificano la loro vita in nome di Allah e per il rischio che sono disposti a correre il loro guadagno è di 10000 euro al mese.

Reclutatori web e in carcere: sono persone che si adattano alla psicologia di chi hanno di fronte. Conducono il prescelto ad una condizione di paranoia e ansia, parlano in continuazione con loro, gli forniscono idee, spunti di pensiero, sia su internet che dal vivo. Adescano con la scusa di poter donare loro solidarietà e comprensione, impongono gli insegnamenti di Al Baghdadi come unica interpretazione dei testi. È presente un certo grado di empatia per entrare in contatto con il prossimo, tuttavia la maschera cade nel momento in cui l'aspirante combattente viene messo alla prova e nel caso decidesse di tornare indietro viene ucciso.

Donne: le schiave dell'Isis (non ci sono diritti per le donne del Daesh) sono coperte dal velo nero in ogni circostanza, vendute come schiave sessuali o date in moglie ai combattenti. Devono obbligatoriamente ubbidire alla Sharia e sono sorvegliate da un reparto speciale della polizia dell'Isis "Al Khansa" in memoria di una poetessa che era cara al profeta Maometto. Prigioniere in casa, con porte rigorosamente chiuse, il loro compito è quello di sostenere i mariti combattenti, educare figli e figlie. Sono vietati tutti i vizi femminili: centri estetici, saloni di bellezza, parrucchieri. Le bambine studiano fino ai 12 anni per poi essere preparate al matrimonio, $i$ bambini anch'essi istruiti ed iniziati alla guerra. Ogni trasgressione è punita con violenze sessuali ripetute, nessuno sconto di pena per le bambine. I "bottini di guerra", come sono soliti chiamare le "donne ostaggio", vengono vendute a prezzi diversi a seconda della loro età e trasformate in concubine, come vuole la jihad. Non esistono dirit- ti per l'infanzia ma ci sono scuole di addestramento chiamate

"Madrasse" in cui il bambino apprende:

- Il Corano ed il monoteismo (Tawhid)

- Giurisprudenza (Fiqh)

- Preghiera (Salat)

- Il credo (Akeeda)

- La vita di Maometto (Hadith e Sura) Orfani, abbandonati dai genitori. Durante il Brainwashing, i bambini vengono privati della paura di morire e mandati ad esplodere con ciondoli ed amuleti portafortuna.

Conoscere ogni aspetto culturale a partire dalla fine dell'epoca dell'ignoranza (pre-islam, Al Jahilittah), aiuta il profiler nel collegamento tra le informazioni. A livello culturale è richiesta la conoscenza della storia di un fenomeno, ad esempio, addentrandosi nella cultura araba si capisce come il jihadismo abbia ereditato determinate caratteristiche: "Arabo" è sinonimo di poeta, cavaliere generoso originario di una cultura tribale in cui la donna era solo una concubina e il modo per poter procreare, non necessariamente l'arabo è musulmano. Lavori umili, conflitti perenni e d esaltazione della morte, poiché con l'arrivo dell'islam e della Jihad (sforzo), la morte in è vita e simbolo d'onore, protetti da Allah come citato nella Sura, verso 169: "Non considerare morti quelli che sono stati uccisi sul sentiero di Allah, sono vivi e ben provvisti dal loro Signore". Per i martiri c'è invece, secondo i testi, la possibilità di salvare 70 persone care dall'inferno. Scuola di pensiero diversa è quella degli sciiti, in attesa del Mahadi (dodicesimo apostolo) e per cui non fanno la jihad (lo sforzo), nonostante dei versi sciiti recitino di abbattere quanti più sunniti possibile per far sì che l'apostolo si manifesti. Nel credo sunnita c'è la forte credenza nella jihad per cui, un atto infedele va punito e incita a combattere gli scorretti. Comune è la visione del paradiso, luogo in cui tutto è concesso e ogni peccato viene spogliato della sua accezione negativa. L'inferno resta il tormento dei ribelli.

L'Isis, proveniente da quest'ultimo culto, fin dallo sfaldamento di Siria e Iraq si è diffuso in regioni dell'Africa in cui domina il caos ed una scorretta vigilanza da parte delle autorità. I flussi sono stati registrati verso: Libia, Tunisia, Somalia e Niger; 1'Italia potrebbe essere un obiettivo per via della posizione prossima al nord Africa; il Kosovo è la Serbia sono tra le mete con più Foreign fighters quindi possibile teatro di scenari futuri, così come i paesi dell'est. In ognuno di questi luoghi in cui manca l'igiene, l'alfabetizzazione e le reti stradali, l'Isis ha provveduto a fornirli guadagnandosi, in parte, il rispetto dei residenti. Sono state offerte: case, vaccini, autostrade. Lo stato del Qatar si rapporta differentemente poiché accusato di finanziare lo Stato Islamico, supposizione smentita tramite Al-Jazeera dall'Imam Izzedin Elzir, presidente dell'Unione delle comunità islamiche italiane. Se il problema Isis è legato ad una cattiva interpretazione del Corano allora è interessante il legame che si crea con la storia e con l'antico califfato ottomano del 1516, legame ricostruito dalla rivista "Dabiq" islamista su ceduta da "Rumiyah". Esse rappresentano la certezza sul corretto approccio alla fede islamica, strumento di legittimazione di un'organizzazione. È ricorrente l'utilizzo dei pronomi e dell'imperativo. Alcuni esempi sono: "Il nostro mondo è sotto minaccia"; "Queste azioni sono in accordo con l'islam"; "È obbligatorio difendere il mondo dalle contaminazioni". Per suscitare terrore nel nemico ecco che si legge quanto segue nei messaggi inviati tramite i media: "Noi siamo a casa vostra". Un messaggio del genere crea paranoia in chi lo ascolta, è una vera e propria minaccia all'invasione della privacy.

I casi studio di contro narrativa riportati qui di seguito sono resi in collaborazione con la Polizia di Stato.

Il primo caso di cui si riporta testimonianza è quello dello spacciatore tunisino indottrinato nelle carceri italiane, il suo reclu- 
tatore viene descritto come un egiziano dalla voce "calda e melodiosa", controllava i nuovi arrivati e li studiava instaurando con loro un dialogo, in particolare le sue vittime, erano quelli instupiditi dalla droga, i soli e poveri. La caratteristica del reclutatore in carcere è quello di riempire le giornate degli altri prigionieri con stimoli ed emozioni nuove. I musulmani, secondo quanto racconta il tunisino vengono raccolti tutti in un'unica sede:" Benvenuto! Ti metto con i tuoi, così vi tenete compagnia tra maomettani" questo è ciò che le guardie dicono loro, sottovalutando il leader occasionale che indottrinava i nuovi arrivati: "Ricordava e raccontava le storie di quando l'islam conquistava il mondo. Chi ci aveva mai raccontato queste cose? Li vedevamo tutti, mi vedevo davanti a loro, mi sentivo esaltato, ispirato, arricchito di minuto in minuto, fa un racconto all'altro. "È bene confrontare la personalità del narratore tunisino con la figura di Lee Oswald l'attentatore del presidente Kennedy in cui si riscontra, come nei terroristi islamici un'anestesia emotiva, in cui l'approccio con il prossimo non include un iter psichico infantile né un'evoluzione affettiva, al contrario si sviluppa un certo grado di aggressività. (Intervista al Prof. Mastronardi sulla figura di Oswald). Secondo esempio con cui si può paragonare lo stato psichico di un terrorista è quello di Dion Terres, mass murder autore della strage in un McDonald nel Wisconsin (USA):

1. Bassa soglia di tolleranza allo stress

2. Depressione

3. Narcisismo.

Questi tre elementi sono tipici sia del mass murder che del terrorista che si approccia al jihadismo. Secondo caso è quello di Hamza, 33 anni ex jihadista che si è tirato indietro nel momento in cui doveva tagliare la gola ad alcuni amici e violentare le schiave del sesso. "Quegli uomini che dicono di combattere per Allah sono solo dei depravati assetati di sangue e sesso." La scelta che Hamza di seguire la Jihad era mossa principalmente da una questione di fede e ripresa dei valori islamici, tuttavia nel momento dell'orrore la sua coscienza ha avuto la meglio sui metodi disumani operati dai suoi ex compagni (Il Messaggero.it, Marzo 2015).

Terzo caso: ex jihadista Tagiko. L'uomo è l'esempio e testimonianza di quali contenuti diffondano le piattaforme virtuali dell'Isis sul web

"Questa è la vera strada, la strada Allah. Dovete uccidere $i$ bambini senza pietà. Dovete uccidere le donne, gli ebrei, $i$ capi degli ebrei, gli infedeli e gli sciiti. Dovete ucciderli tutti"

(Il Timone, Aprile 2017).

Come ultimo caso si riporta la testimonianza di una donna partita per servire il califfato ma che si è ritrovata in un incubo, si tratta di Aisha. La ragazza d'origine olandese si è pentita di aver seguito il suo fidanzato Yilmaz, combattente dell' Isis ed è stata salvata e portata indietro da sua madre che senza esitare e con coraggio è partita per la Siria per salvare sua figlia (NanoPress.It, Settembre 2014).

L'approccio della narrativa proposto dagli esperti del settore diviene funzionale se applicata in concomitanza ai poteri delle forze dell'ordine nel rispetto della giurisdizione. La scelta della risoluzione migliore scaturisce dalla tipologia di profilo che si sta esaminando, per questo, le tabelle proposte in tale sede sono lo strumento necessario ad un corretto svolgimento delle fasi di analisi adattandosi a qualunque situazione. Il crimine è cambiato, e l'arma per il contrasto è cambiata con lui. In conclusione, ciò che si vuole dimostrare attraverso il presente lavoro è che esiste la possibilità, se non di eliminare, di ridurre la minaccia jihadista avendo buone probabilità di riuscita, qualora il fenomeno prendesse piede sotto nuove spoglie.

\section{Bibliografia}

Arquilla J., Ronfeldt D., (2001), Networks and Netwars: The future of terror, crime, and militancy. National Defense Research Institute RAND, pag. 7-8.

Belvedere R., (2011), Dietro i media del Quarto Mondo. Nuova geopolitica della comunicazione, Ed. Armando Armando, Roma.

Bertolotti C., (2020), Correlazione tra flussi migratori e minaccia riconducibile al terrorismo di matrice confessionale, tipografia del Centro Alti Studi Strategici, Roma.

Bianchi P., Carbone M, Vallefuoco V., (2020), IPSOA Guide Operative: Le nuove regole antiriciclaggio, Wolters Kluwer Editore, Milano.

Braddock K., (2020), Weaponized words, The strategic Role of Persuasion in Violent Radicalization and CounterRadicalization, Cambridge University Press.

Calderaro M.,(2019-2020), Profiling e analisi comportamentale, Istituto Internazionale Scienze Criminologiche e Psicopatologico Forensi, Roma.

Freedman J.L., Fraser S.C., (1966), Compliance without pressure: the foot-in-the-door technique. in Journal of personality and Social Psychology.

Gibson W., (1984), Neuromancer, prima edizione, Orion Publishing Co, UK.

Khan R.L., Cannell C.F., (1968), Le dinamiche dell'intervista, Marsilio Editore, Venezia.

Lapi M., (2018), Tecniche di analisi strutturata, edizioni Themis, Roma.

Mastronardi V., Palermo G., (2005), Il profilo criminologico, dalla scena del crimine ai profili socio-psicologici, Giuffrè editore, Milano.

Mastronardi V., (2006), Sette sataniche. Dalla stregoneria ai messaggi subliminali nella musica rock, dai misteri del mostro di Firenze alle Bestie di Satana. Newton Compton Editori.

Mastronardi V., Leo S., (2007), I terroristi. Dalle Brigate Rosse all'IRA, dal terrorismo basco al terrorismo turco, dal pkk al terrorismo ceceno, fino ad arrivare alla temutissima Al-Qaeda, Newton Compton, Milano.

Mastronardi V., (2013), Le strategie della comunicazione umana: la persuasione, le influenze sociali, i mass media, quarta edizione, FrancoAngeli editore.

Matronardi V., (2016), Manuale di comunicazione non verbale, Carocci Faber Editori, Roma.

Mastronardi V., (2017), Prevenzione alla radicalizzazione, in Progetto Mates.

Ministero della Giustizia (DAP) (giugno 2012) La radicalizzazione del terrorismo islamico. Elementi per uno studio del fenomeno di proselitismo in carcere Quaderni ISSP n. 9, Istituto Superiore di Studi Penitenziari.

Monzani M., Benatti F., (2015), Criminologia, psicologia investigativa e psicopedagogia forense. libreriauniversitaria.it Edizioni, Padova.

Plebani D., (2019), Da Raqqa a Boghuz: l'evoluzione di Stato Islamico tra il 2017 e il 2019, Associazione Italiana Editori, Milano.

Scipione L, (2017), Dal terrorismo politico alle nuove forme di terrorismo globale: strumenti di conoscenza e di contrasto in ambito nazionale ed europeo, SNA scuola nazionale dell'amministrazione.

Strano M., (2007), Manuale di investigazione criminale, NSTecna Editore, Roma.

Teti A., (2018), Cyber espionage e cyber counterintelligence, Rubettino Editore.

Vidino L., (2015), L'Italia e il terrorismo in casa: che fare? Epoké Edizioni.Milano. 


\section{Sitografia}

Acquaviva M., (2020), Interrogatorio penale: come funziona? In la legge per tutti.it. https://www.laleggepertutti.it/362185 interrogatorio-penale-come-funziona

Adescamento online: che cos'è e come riconoscerlo, (2020), in Save the children. https://www.savethechildren.it/blog-notizie/adescamento-online-che-cos-e-come-riconoscerlo

Annicchiarico G., (2019) Rimpatri fantasma, l'Europa non vuole i suoi miliziani, Il Manifesto. https://ilmanifesto.it/rimpatri-fantasma-leuropa-non-vuole-i-suoi-miliziani/

Barone D., (2019), La digitalizzazione del califfato e le nuove forme di conflitto di daesh, in Cesi-Italia.org. https://cesi-italia.org/articoli/1009/la-digitalizzazione-del-califfato-e-lenuove-forme-di-conflitto-di-daesh

BBC News, (2018), Fouad Belkacem: Belgian Islamist leader loses citizenship, in bbc.com. https://www.bbc.com/news/ world-europe-45951138

Belladonna F., (2017), Global Terrorism Index: meno morti per terrorismo, ma aumentano i Paesi colpiti, in asvis.it. https://asvis.it/goal16/notizie/491-1115/global-terrorism-indexmeno-morti-per-terrorismo-ma-aumentano-i-paesi-colpiti-

Bertolotti C., (2020), Correlazione tra flussi migratori e minaccia riconducibile al terrorismo di matrice confessionale, tipografia del Centro Alti Studi Strategici, Roma. http://www.difesa.it/ SMD_CASD/IM/CeMiSS/Pubblicazioni/ricerche/Pagine/AO_ CC_01.aspx

Canali L., (2016), Il Belgio dei Jihadisti, in limes online.com. https://www.limesonline.com/il-belgio-dei-jihadisti/90573

Cannavicci M., (2006), Metodologia e tecnica dell'interrogatorio, in Atti e Modelli di Polizia Giudiziaria.eu. https://attiemodellidipoliziagiudiziaria.eu/files/L-INTERROGATORIO.pdf

Cannavicci M., Psicologia del terrorismo, in CEPIC (Centro europeo di psicologia investigazione e criminologia). https://view.officeapps.live.com/op/view.aspx?src=http $\% 3 \mathrm{~A} \%$ 2F\%2Fcepic-psicologia.it\%2Fcontributi\%2FPSICOLOGIA\%2520DEL\%2520TERRORISMO.doc

Cannavicci M., (2017), Verso un approccio italiano alla prevenzione della radicalizzazione, in Commissione studio su fenomeno della radicalizzazione e dell'estremismo jihadista.

Carbonelli M., (2018), Terrorismo: dalle definizioni internazionali alle Condotte di reato, in Safety\&Security magazine. https://www.safetysecuritymagazine.com/articoli/terrorismodalle-definizioni-internazionali-alle-condotte-di-reato/

Cecchini R., (2015), Che cos'è il Dark Web?, in GarrNews.it. https://www.garrnews.it/index.php/ricerche/464

Colquhoun C., (2016), Inghimasi -The Secret ISIS Tactic Designed for the Digital Age, in bellingcat.com. https://www. bellingcat.com/news/mena/2016/12/01/inghimasi-secret-isistactic-designed-digital-age/

Commissione parlamentare per l'infanzia, (1991), Ratifica ed esecuzione della convenzione sui diritti del fanciullo, fatta a New York il 20 novembre 1989. https://www.camera.it/_bicamerali/infanzia/leggi/1176.htm

Cruciati C.,(2019), Nei campi di detenzione in Siria c'è L'isis "Invisibile", Il Manifesto. https://ilmanifesto.it/nei-campi-didetenzione-in-siria-ce-lisis-invisibile/

Delicato F., (2015), criminiseriali.it http://www.criminiseriali.it/ Terrorismo $\% 20 \mathrm{e} \% 20$ Internet.html

Dworkin A., (2019) Beyond good and evil: Why Europe should bring ISIS foreign fighters home, in European Council on Foreign Relationsecfr.eu. https://www.ecfr.eu/page/-/beyond good_and_evil_why_europe_should_bring_isis_foreign_fighters_home.pdf

Fraser S.C., Freedman J.L., (1966), Compliance without pressure: the foot-in-the-door technique. http://bulidomics.com/w/images/6/6c/Freedman_fraser_footinthedoor_jpsp1966.pdf

Giuliani I., (2016), Il profilo psicologico del combattente e del kamikaze Isis, in affaritaliani.it. https://www.affaritaliani.it/ blog/criminalmente/il-profilo-psicologico-del-combattentedel-kamikaze-isis-427408

Henningan W. J., (2019), Abu Bakr al Baghdadi is dead. Where does that leave ISIS?, in Time, 2019. https://time.com/ 5711828/al-baghdadi-dead-isis-future/

Irrente K., (2015), I pentiti dell'Isis: storie di occidentali arruolati coi jihadisti che ci hanno ripensato e vogliono tornare a casa, in nanopress.it. https://www.nanopress.it/storie/mondo/i-pentiti-dellisis-storie-di-occidentali-arruolati-coi-jihadisti-che-cihanno-ripensato-e-vogliono-tornare-a-casa/48967

Kubark, (1963) Kubark counterintelligence interrogation, Top Secret document, approved for release in 1997. https://nsarchive2.gwu.edu/NSAEBB/NSAEBB27/docs/doc01.pdf

Lodini C., Serafini M., (2015), I disertori del Califfato "Scappiamo per non uccidere in nome di Allah", in Il Corriere della Sera. http://reportage.corriere.it/esteri/2015/i-disertori-del-califfatolasciamo-perche-non-sopportiamo-piu-lorrore/

Lorusso F., (2017), Rojava il fiore del deserto. Intervista a Davide Grasso, combattente YPG in Siria, in Carmillaonline. https://www.carmillaonline.com/2017/09/05/rojava-fiore-neldeserto-intervista-davide-grasso-combattente-ypg-guerrasiria-rivoluzione/

Macagnone F., (2015),Isis, ex jihadista racconta lo Stato Islamico: "Volevano sgozzassi i miei amici e stuprassi giovani yazidi", in ilmessaggero.it. https://www.ilmessaggero.it/primopiano/esteriisis_ex_jihadista_racconta_omicidi_stupri-926591.html

Marone F., (2019), Dopo il jihad, profilo di un foreign fighter disilluso, in ISPI online. https://www.ispionline.it/it/pubblicazione/dopo-il-jihad-profilo-di-un-foreign-fighter-disilluso-22536

McKenna A., (2019) Boko Haram Nigirian Islamic Group, in Britannica. https://www.britannica.com/topic/Boko-Haram

Orsini A., (2019), Turchia: il rimpatrio dei jihadisti dell'ISIS inizierà l'11 novembre, in sicurezza internazionale, quotidiano di informazione. https://sicurezzainternazionale.luiss.it/2019/11/ 10/turchia-rimpatrio-dei-jihadisti-dellisis-iniziera-111novembre/

Piazza S., (2018), Quel che resta di Sharia4Belgium in Europa, in babilomagazine.it https:/www.babilonmagazine.it/sharia4belgium-fouad-belkacem/

Planas B., (2020), Dipendenza da internet: sintomi, casi, conseguenze, a chi rivolgersi. https://www.psicologabeatriceplanas.it/tag/ casi-di-dipendenza-da-internet

Puente D., (2019), TikTok, rimossi gli account Isis: promuovevano la jihad attraverso video di esecuzioni con musica e cuoricini, in Open.online. https://www.open.online/2019/10/24/tiktok-rimossi-gli-account-isis-promuovevano-la-jihad-attraverso-video-diesecuzioni-con-musica-e-cuoricini/

sapere.it, Che cos'è il Cyberspazio. De Agostini Editore. https://www.sapere.it/sapere/strumenti/domande-risposte/ditutto-un-po/definizione-cyberspazio.html

Sgamotta A., (2015), Belgio, l'irresistibile ascesa del jihad nel cuore dell'Europa, in linkiesta.it. https://www.linkiesta.it/2015/11/belgio-lirresistibile-ascesa-del-jihad-nel-cuore-delleuropa/

Signorelli A.D., (2019), La propaganda dell'isis adesso passa da TikTok, in Lastampa.it. https://www.lastampa.it/tecnologia/idee/ 2019/10/27/news/la-propaganda-dell-isis-adesso-passa-da-tiktok-1.37793613

Stefanini M., (2019), Il Belgio fa i conti con il separatismo degli immigrati e l'integralismo islamico, in l'incontro.news. https://www.lincontro.news/il-belgio-fa-i-conti-con-il-separatismo-degli-immigrati-e-lintegralismo-islamico/

The House of Representatives, Department for Public and 
International Relations, (2004), Brussels. https://web.archive. org/web/20051112001107

The Meir Amit Intelligence and Terrorism Information Center, (2019) Isis's Media Network: Developments in 2018 and future courses of action, in terrorism-info.org. https://www.terrorism-info.org.il/en/isiss-media-network-developments-2018future-courses-action/

Ummah, in Oxdorf islamic studies online dictionary http://www. oxfordislamicstudies.com/article/opr/t125/e2427

Vitale I., (2016), La tecnica di interrogatorio Reid spiegata passo per passo, in igorvitale.org. https://www.igorvitale.org/la-tecnica-di-interrogatorio-reid-spiegata-passo-per-passo/

Zabbeo N., (2018), Navigazione anonima, Darknet e VPN: soluzioni e consigli per l'accesso sicuro a internet, in cybersecurity360.it. https://www.cybersecurity360.it/soluzioni-azienda- li/navigazione-anonima-darknet-e-vpn-soluzioni-e-consigliper-laccesso-sicuro-a-internet/

\section{Filmografia}

Criminal Minds, episodio 2x10, L'ora della preghiera, 2006.

InsiderOver, Il Jihadista che parla italiano, 2017. https://youtu.be/A7FW2iuXR_gNomadetv24

Jihadista europeo in Siria, 2015. https://youtu.be/W-dWIIrqDOk

Piazza Pulita. Noi Jihadisti arriveremo a Roma, 2015. https://youtu.be/g0nnsVG-EWk

Piazza Pulita. Sono un soldato dell'Isis, 2017 https://youtu.be/ F4Wxb05TYE
Correspondence: Maria Campioni.

E-mail: mariacampioni8@gmail.com

Key words: criminological profile; Islamic terrorist Parole chiave: profilo criminologico; terrorista islamico. Palabras clave: perfil criminológico; terrorista islámico.

Received for publication: 19 March 2020.

Accepted for publication: 8 May 2020

This article is distributed under the terms of the Creative Commons Attribution Noncommercial License (by-nc 4.0) which permits any noncommercial use, distribution, and reproduction in any medium, provided the original author(s) and source are credited.

${ }^{\circ}$ Copyright: the Author(s), 2020

Licensee PAGEPress, Italy

Rivista di Psicopatologia Forense, Medicina Legale, Criminologia 2020; $25: 543$

doi:10.4081/psyco.2020.543 